\title{
Biomimetic Design of a Robustly Stabilized Folded State Enabling Seed-initiated Helical Supramolecular Polymerization under Microfluidic Mixing
}

\author{
Soichiro Ogi, ${ }^{* \dagger}$ Aiko Takamatsu, ${ }^{\dagger}$ Kentaro Matsumoto, ${ }^{\dagger}$ and Shigehiro Yamaguchi $*, \dagger, *$ \\ 'Department of Chemistry, Graduate School of Science, and Integrated Research Consortium on Chemical Science (IRCCS), \\ Nagoya University, Furo, Chikusa, Nagoya 464-8602, Japan \\ Institute of Transformative Bio-Molecules (WPI-ITbM), Nagoya University, Furo, Chikusa, Nagoya 464-8602, Japan \\ Helical supramolecular polymers, Amino acids, Diamide folding, Out-of-equilibrium systems, Seeded polymerization
}

\begin{abstract}
We have investigated the folding and assembly behavior of an alanine-based monomeric diamide and a cystine-based dimeric diamide bearing pyrene units and solubilizing alkyl chains at their C-termini and N-termini, respectively. In low-polarity solvents, the former molecule forms a folded 7-membered ring conformation with an intramolecular hydrogen bond, while the latter molecule forms a 14-membered ring through double intramolecular hydrogen bonds between two diamide units. Spectroscopic studies revealed that both folded states are thermodynamically unstable and eventually transform into more energetically stable supramolecular polymers. Importantly, compared to the alanine-based diamide, the cystine-based dimeric diamide exhibits a superior kinetic stability in the metastable folded state, as well as an increased thermodynamic stability in the aggregated state. Consequently, spontaneous transformation from the folded state into the aggregated state is retarded even under the conditions of rapid molecular diffusion. Accordingly, the initiation of supramolecular polymerization can be regulated via a seeding approach under microfluidic mixing conditions. Furthermore, the supramolecular polymer composed of the cystine-based dimeric diamide has a helical structure with an enhanced chiral excitonic coupling between the transition dipoles of the pyrene units. Taking advantage of the self-sorting behavior observed in a mixture of L-cysteine- and D-cysteine-based dimeric diamides, a two-step supramolecular polymerization from a racemic mixture was achieved by stepwise addition of the corresponding seeds.
\end{abstract}

\section{INTRODUCTION}

Amyloid fibrils are a class of supramolecular polymers found in biological systems and consist of highly ordered onedimensional aggregates of polypeptide chains. ${ }^{1,2}$ In native proteins, the polypeptides inherently form an $\alpha$-helix conformation via a folding process dominated by intramolecular interactions. However, misfolding occasionally occurs to produce $\beta$-sheet conformations, which interact intermolecularly with one another to form amyloid fibrils via a nucleation-elongation mechanism. ${ }^{3-8}$ The rate of the nucleation is low because misfolding of the protein involves multiple rearrangements, while elongation proceeds quickly due to the favorable intermolecular interactions between the misfolded polypeptides. Importantly, there is a significant lag time before initiation of the elongation, during which the external addition of aggregates as seeds can accelerate the formation of amyloid fibrils from metastable native proteins. ${ }^{9-13}$

Similar seeding effects have also been observed in the selfassembly of synthetic molecules with a $\pi$-conjugated core, in which a nucleation-elongation process competes with a folding process. ${ }^{14-16}$ Such seed-initiated polymerization can be an attractive method to prepare one-dimensional aggregates with controlled structural parameters, such as average size, polydispersity, $^{17-21}$ and molecular sequence. ${ }^{22-28}$ The use of hydrogen bonds is an effective way to establish a controlled self-assembly process, as it offers high designability of both intermolecular and intramolecular interactions. ${ }^{14,29}$ The intermolecular interactions guide the self-assembly of molecules by regulating their molecular arrangement in the aggregated state. Intramolecular interactions can produce specific folded structures in the non-aggregated state. The kinetic folding of a monomer into a conformation different from its aggregated state conformation can retard its spontaneous participation in nucleation-growth supramolecular polymerization. Such non-equilibrium supramolecular polymerization has been achieved for amide-substituted $\pi$ conjugated molecules with a bowl-shaped $\pi$-skeleton ${ }^{30}$ or hydrogen-bond acceptor units. ${ }^{31-33}$ Amide groups connected to an additional amide, ${ }^{34}$ ester, $^{35,36}$ or 1,2,3-triazole group ${ }^{37}$ by a short alkyl linker also enable kinetic control over the selfassembly of $\pi$-conjugated molecules via the formation of intramolecular hydrogen bonds.

We have previously reported an amino-acid-based diamide that is a versatile structural motif for seed-initiated supramolecular polymerization (Figure 1a). ${ }^{38}$ When the diamide was cooled in low-polarity solvents, the equilibrium favored a folded conformation with a seven-membered ring via an intramolecular hydrogen bond (Figure 1b). In this state, supramolecular polymerization through intermolecular hydrogen bonding interactions was temporarily retarded. As this folded inactive state was thermodynamically unstable, it eventually transformed into a more thermodynamically stable aggregated state. Importantly, initiation of the supramolecular 
a

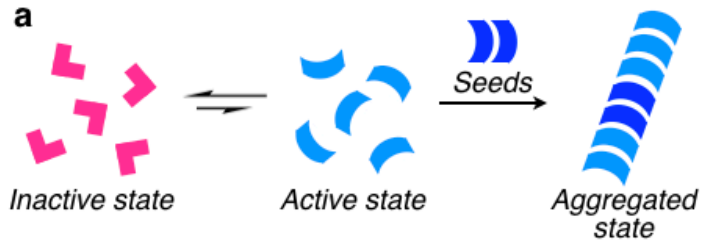

b

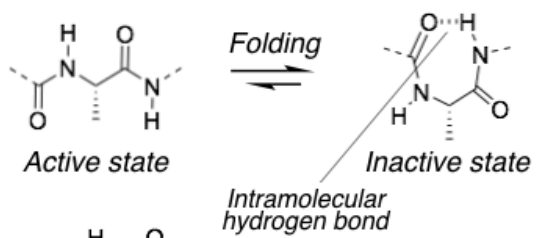

c

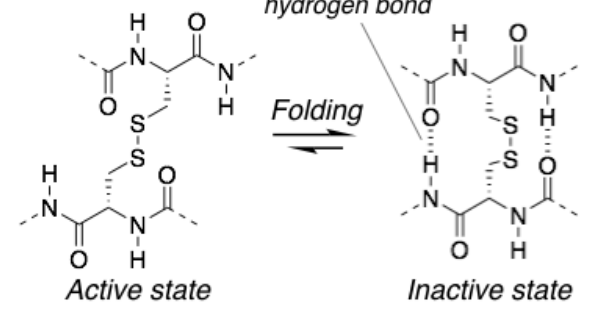

d
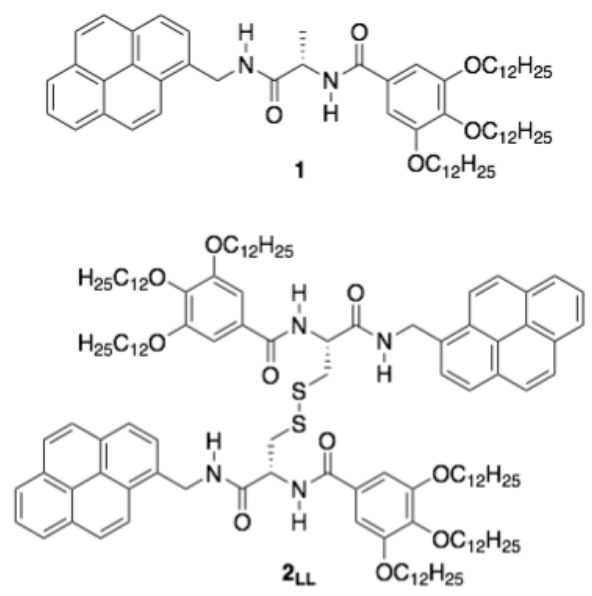

Figure 1. (a) Schematic representation of seeded polymerizations; the folding processes of (b) L-alanine-based diamide and (c) Lcysteine-based dimeric diamide, and (d) the chemical structures of 1 and $\mathbf{2}_{\mathrm{LL}}$.

polymerization was successfully regulated by the external addition of seeds. A further challenge in this chemistry is the production of a stiffer folded state that could persist even under stirring or flowing conditions. ${ }^{39-41}$ Such mechanical agitation is required for the mass production of controlled supramolecular polymers in order to achieve homogeneous and rapid mixing of the seeds with the metastable monomers and increase the subsequent elongation rate of the seeds. However, folding based on a single intramolecular hydrogen bond is not sufficient to stabilize the metastable state. The rational design of a robustly stabilized metastable state would increase the utility of seeded supramolecular polymerization.

Extensive researches into amyloid fibrils has shown that the supramolecular polymerization of misfolded proteins is proceeded by a significant lag time. ${ }^{7}$ An important implication of this fact is that the conversion from a native conformation to a misfolded one proceeds through a large number of steps involving the rearrangement of multiple intramolecular interactions. Thus, increasing the number of intramolecular hydrogen bonding interactions should be a key strategy in the molecular design of amino-acid-based diamide systems with a kinetically stabilized folded state (Figure 1c). To fulfill this requirement, we designed a dimeric amino-acid-based diamide with a disulfide linkage. To study the efficacy of this molecular design, we synthesized alanine-based diamide $\mathbf{1}$ and L-cysteinebased dimeric diamide $\mathbf{2}_{\mathrm{LL}}$, in which pyrene units and solubilizing alkyl chains are introduced at the C-termini and $\mathrm{N}$ termini, respectively (Figure 1d). The introduction of pyrene units should facilitate monitoring of the folding and aggregation processes via changes in the UV-vis absorption and emission properties. In-depth study of their folding and aggregation properties revealed that dimerization of the amino-acid-based diamide skeleton not only enhances the thermodynamic stability of the aggregated state, but also increases the kinetic stability of the metastable folded state. Accordingly, the nucleation-growth supramolecular polymerization was preceded by a significant lag time, and thereby, we were able to kinetically regulate the initiation step of the supramolecular polymerization using a seeding method even under microfluidic mixing conditions.

\section{RESULTS AND DISCUSSION}

Synthesis. Alanine-based diamide 1 was synthesized in two steps from $N$-(tert-butoxycarbonyl)-L-alanine by amidation with 1-pyrenylmethylamine, followed by $N$-Boc deprotection and amidation of the $\mathrm{NH}_{2}$ terminus by treatment with 3,4,5tris(1-dodecyloxy)benzoic acid. Pyrenyl-substituted dimeric diamide $\mathbf{2}_{\mathbf{L L}}$ was prepared in three steps from $N$-[ $(9 \mathrm{H}-$ fluoren9-ylmethoxy)carbonyl]-S-(triphenylmethyl)-L-cysteine. Thus, the $\mathrm{COOH}$ terminus of the $\mathrm{L}$-cysteine derivative was amidated with 1-pyrenylmethylamine. $N$-Boc deprotection and amidation of the $\mathrm{NH}_{2}$ terminus were then conducted by treatment with 3,4,5-tris(1-dodecyloxy)benzoic acid. Subsequent deprotection of the $S$-trityl protecting group directly produced disulfide $\mathbf{2}_{\mathbf{L L}}$ under oxidative deprotection conditions. The details of the syntheses and characterizations are described in the Supporting Information.

Folding. Initially, the folding behavior of $\mathbf{1}$ and $\mathbf{2}_{\mathrm{LL}}$ was investigated using ${ }^{1} \mathrm{H}$ NMR spectroscopy. The signal of the amide proton adjacent to the pyrenyl group in $\mathbf{1}$ was observed at $6.59 \mathrm{ppm}$ in chloroform- $d_{1}$, which represented a downfield shift of $0.93 \mathrm{ppm}$ compared to that of our previously reported pyrenyl-substituted monoamide (Figure S1 in Supporting Information). ${ }^{38}$ This can be explained by the folding of the diamide moiety in a seven-membered ring fashion with an intramolecular hydrogen bond. In contrast, $\mathbf{2}_{\mathrm{LL}}$ exhibited a proton signal at $9.17 \mathrm{ppm}$, which was assigned to the amide protons adjacent to the pyrenyl group based on twodimensional NMR spectroscopy (Figures S2 and S3). Importantly, this proton signal was shifted significantly downfield $(2.58 \mathrm{ppm})$ compared to that of $\mathbf{1}$. Its chemical shift exhibited a slight concentration dependence at concentrations from $5 \times 10^{-4} \mathrm{M}$ to $2 \times 10^{-3} \mathrm{M}$ (Figure S4). Thus, the diamide moieties in $\mathbf{2}_{\mathrm{LL}}$ preferentially form intramolecular hydrogen bonds with a conformation different from that of $\mathbf{1}$.

To elucidate the folded conformation of $\mathbf{2}_{\mathrm{LL}}$, we conducted DFT calculations using the model compound $\mathbf{2}^{\prime}$, in which the dodecyloxy chains in $\mathbf{2}_{\mathbf{L L}}$ were replaced with methoxy groups 

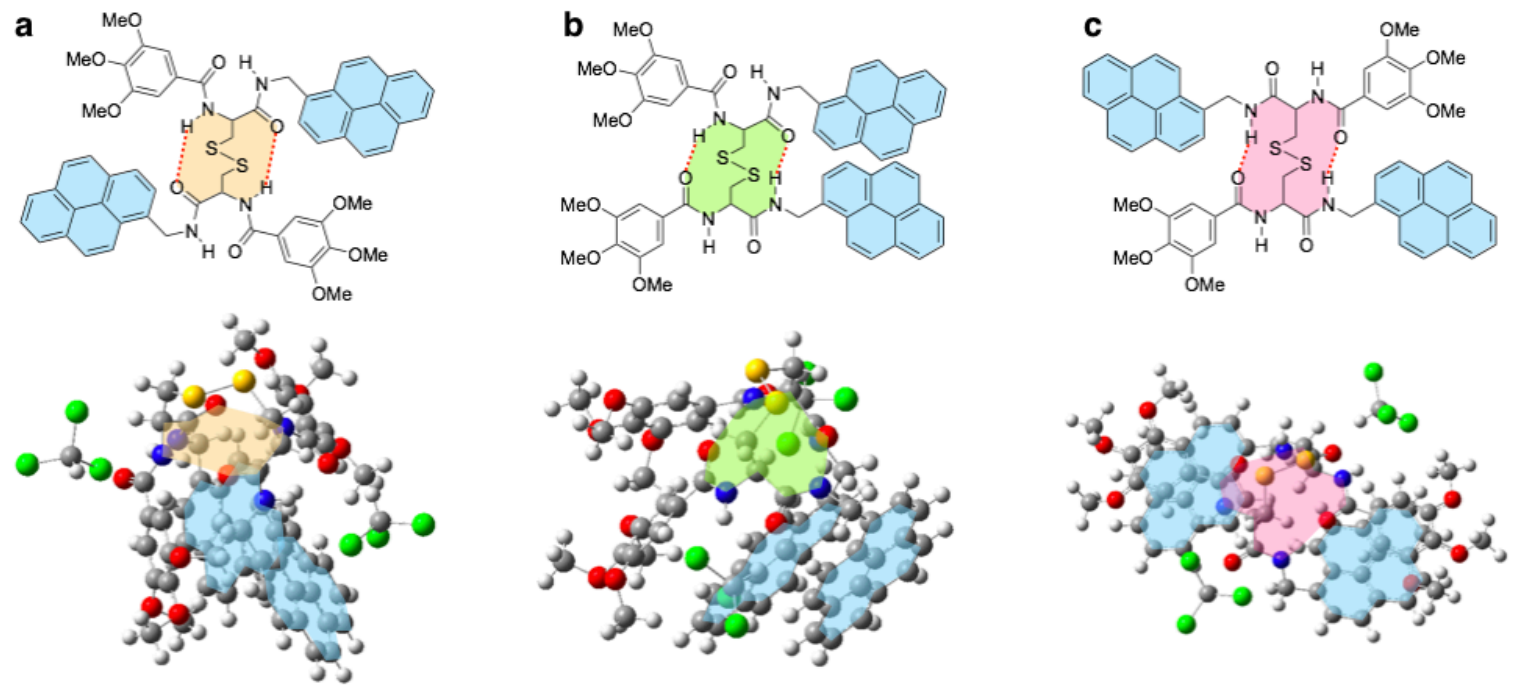

Figure 2. Chemical structures and geometries of 2' with a (a) 10-membered, (b) 12-membered, or (c) 14-membered ring of hydrogen bonding network containing $\mathrm{CHCl}_{3}$ molecules, as optimized using DFT with the $\mathrm{M} 052 \mathrm{X} / 6-31 \mathrm{G}(\mathrm{d})$ basis

for simplicity. Three types of intramolecular hydrogen-bonded networks, namely, 10-membered, 12-membered, and 14membered ring-based structures, were considered as possible conformations (Figure 2). These structures were optimized at the M052X/6-31G(d) level of theory by including two chloroform molecules, which were placed near the amide groups. The calculations revealed that the 14-membered-ring hydrogen-bonded conformation was more energetically stable than the 10-membered-ring and 12-membered-ring-based conformations by $6.1 \mathrm{~kJ} \mathrm{~mol}^{-1}$ and $4.7 \mathrm{~kJ} \mathrm{~mol}^{-1}$, respectively. NMR calculations using the optimized 14-membered-ring structure were conducted at the B3LYP/6-311+G(2d,p) level. The calculated chemical shifts of the amide protons were 6.93(5) ppm and 9.41(6) ppm, which were in good agreement with the experimentally observed chemical shifts (Table S1). These results indicate that the 14-membered-ring structure is the most feasible folded conformation for $\mathbf{2}_{\mathbf{L L}}$ in the nonaggregated state.

A characteristic feature of the 14-membered-ring folded conformation in $\mathbf{2}_{\mathbf{L L}}$ is that two pyrene units are located far from each other, with a distance of ca. $0.9 \mathrm{~nm}$ between them (Figure $2 c)$. This is in contrast to the folded conformations with 10 membered or 12-membered rings, in which the two pyrene planes are closely aligned within $0.4 \mathrm{~nm}$ (Figures 2a and 2b). Our previous study of two pyrenyl-substituted alanine-based diamides showed that the folded conformation of the diamide moiety in which the two pyrenyl groups were in close proximity resulted in the emergence of a characteristic excimer emission of the pyrene moieties. ${ }^{38}$ Thus, the fluorescence spectrum of $\mathbf{2}_{\mathbf{L L}}$ was examined to obtain information regarding the interaction between the pyrene units in its folded conformation. In chloroform, $\mathbf{2}_{\mathbf{L L}}$ exhibited a monomer emission band with a maximum $\left(\lambda_{\mathrm{em}}\right)$ at $396 \mathrm{~nm}$, indicating the absence of communication between the two pyrene moieties (Figure 3, solid line). Upon addition of the hydrogen-bond-acceptor dimethyl sulfoxide (DMSO) to the chloroform solution of $\mathbf{2}_{\mathbf{L L}}$, an excimer emission band emerged with the $\lambda_{\mathrm{em}}$ at $479 \mathrm{~nm}$ (Figure 3, dashed lines). In the presence of DMSO, the equilibrium is likely shifted from the intramolecularly hydrogen-bonded conformation to a different conformation, resulting in the emergence of the excimer emission. A ${ }^{1} \mathrm{H}$ NMR spectroscopic study also confirmed the effect of DMSO on the intramolecular hydrogen bonds in the folded conformation. Upon addition of DMSO- $d_{6}$ to a solution of $\mathbf{2}_{\mathbf{L L}}$ in chloroform$d_{1}$, an upfield shift of 0.44 ppm was observed for the amide protons adjacent to the pyrenyl groups (Figure S5).
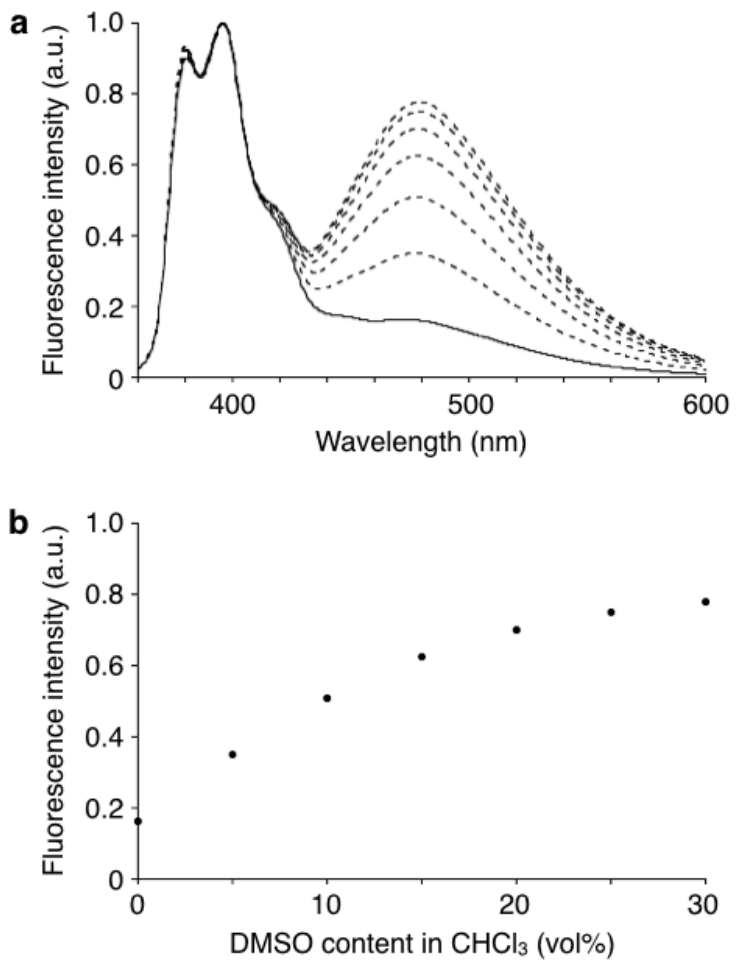

Figure 3. (a) Change in the fluorescence spectra of $\mathbf{2}_{\mathbf{L L}}$ in chloroform with the addition of DMSO, and (b) plot of the ratio of the fluorescence intensity at 396 and $479 \mathrm{~nm}\left(I_{479} / I_{396}\right)$ as a function of the DMSO content in chloroform. 
Aggregation. The monomeric diamide $\mathbf{1}$ and dimeric diamide $\mathbf{2}_{\mathbf{L L}}$ formed aggregates in nonpolar methylcyclohexane $(\mathrm{MCH})$. Their self-assembly behavior was monitored using UV absorption spectroscopy. For sample preparation, each compound was dissolved in $\mathrm{MCH}$ at a concentration of $5 \times$ $10^{-5} \mathrm{M}$ at $363 \mathrm{~K}$ and then cooled to $293 \mathrm{~K}$ at a rate of $1 \mathrm{~K}$ $\min ^{-1}$. In the spectrum of monomeric diamide 1 , an absorption band with a characteristic vibronic structure corresponding to monomeric pyrene and an absorption maximum $\left(\lambda_{\text {abs }}\right)$ at 343 $\mathrm{nm}$ was observed immediately after cooling the solution to 293 $\mathrm{K}$ (Figure S6a), which indicated that $\mathbf{1}$ existed in a nonaggregated form at this stage. While the absorbance at $343 \mathrm{~nm}$ remained unchanged for a few hours, it gradually decreased in intensity, accompanied by the appearance of a new band with the $\lambda_{\text {abs }}$ of $355 \mathrm{~nm}$ (Figure 4 and S6a). This spectral change was attributed to the emerging interaction between pyrene moieties in the ground state. The formation of fibrous aggregates was confirmed using transmission electron microscopy (TEM) (Figure S6b). The Fourier transform infrared (FTIR) spectrum of aggregated 1 exhibited an N-H stretching band at 3270 $\mathrm{cm}^{-1}$, indicating intermolecular interaction between the amide moieties (Figure S6c). These results demonstrated that the nonaggregated diamide $\mathbf{1}$ formed upon cooling is a metastable state, while the supramolecular polymer is thermodynamically stable due to the intermolecular $\pi$-stacking and hydrogen-bonding interactions between the pyrene units and the diamide groups, respectively. Dimeric diamide $\mathbf{2}_{\mathbf{L L}}$ also showed spontaneous self-assembly behavior under the same conditions to form supramolecular polymers; the greatest difference between $\mathbf{1}$ and $\mathbf{2}_{\mathbf{L L}}$ was the longer lag time ( $>12$ hours) for the initiation of the self-assembly of $\mathbf{2}_{\mathbf{L L}}$ (Figures 4 and S6d). The increased lag time implies that the kinetic stability of $\mathbf{2}_{\mathbf{L L}}$ in the nonaggregated state is enhanced by the multiple intramolecular hydrogen-bond interactions in its folded form.

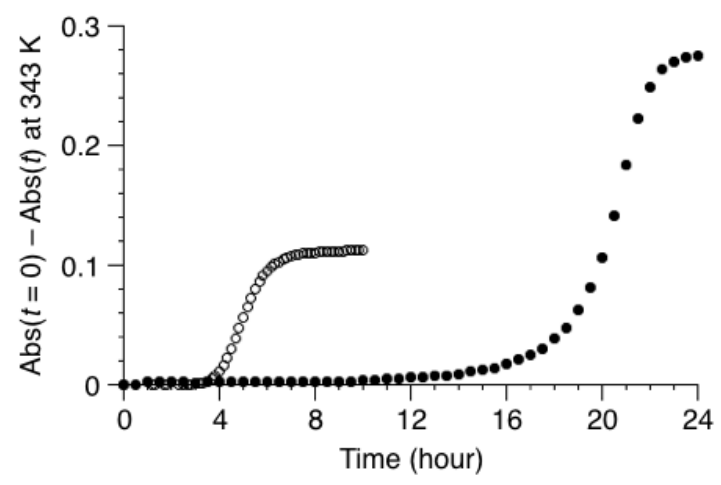

Figure 4. Time courses of the absorbance changes at $343 \mathrm{~nm}$, which correspond to the spontaneous supramolecular polymerizations of $\mathbf{1}$ (open circles) and $\mathbf{2}_{\mathrm{LL}}$ (closed circles) in methylcyclohexane $\left(5 \times 10^{-5} \mathrm{M}\right)$ after fast cooling from $363 \mathrm{~K}$ to $293 \mathrm{~K}$.

The spontaneous transition from the non-aggregated state into the aggregated state was accelerated and completed within 10 min when sonication was applied to a hot MCH solution of molecularly dispersed $\mathbf{2}_{\mathrm{LL}}$ in a water bath below $283 \mathrm{~K}$. The resultant solution showed a UV absorption band corresponding to the aggregated state at $354 \mathrm{~nm}$ (Figure 5a). The circular dichroism (CD) spectra of the aggregated $\mathbf{2}_{\mathbf{L L}}$ showed a CD absorption band with a positive maximum at $357 \mathrm{~nm}$. The CD intensity of $\mathbf{2}_{\mathrm{LL}}$ was greater than that of $\mathbf{1}$ in the aggregated state, demonstrating the larger chiral excitonic coupling between transition dipoles of the pyrene moieties in the aggregated $\mathbf{2}_{\mathbf{L L}}$ (Figure 5b, solid line). When D-cysteine was employed to synthesize the dimeric amino-acid-based diamide $\mathbf{2}_{\mathbf{D D}}$, the CD spectrum of its aggregated state was a perfect mirror image of that of aggregated $\mathbf{2}_{\mathbf{L L}}$ (Figure $5 \mathrm{~b}$, dashed line). The mirrored CD spectra showed that the chiral centers of dimeric diamide 2 substantially impact the arrangement of the pyrene moieties in the supramolecular polymer chains. Atomic force microscopy (AFM) images confirmed that the supramolecular polymers of $\mathbf{2}_{\mathbf{L L}}$ formed left-handed helices with a unimolecular width of 1$2 \mathrm{~nm}$, while those of $\mathbf{2}_{\mathrm{DD}}$ in the aggregated state exhibited the opposite helicity (Figures S7 and S8).
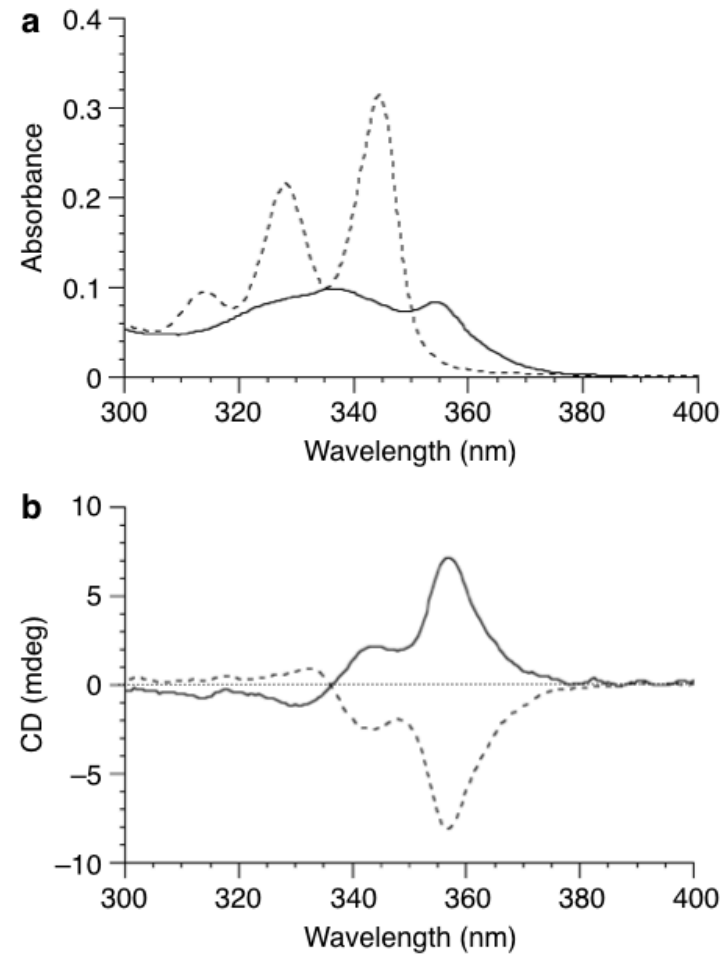

Figure 5. (a) UV absorption spectra of $\mathbf{2}_{\mathrm{LL}}$ in the non-aggregated state (dashed line), obtained after slow cooling at $1 \mathrm{~K} \mathrm{~min}^{-1}$, and in the aggregated state (solid line), obtained after sonication for 10 min in $\mathrm{MCH}\left(4 \times 10^{-5} \mathrm{M}\right)$, and (b) CD spectra of $\mathbf{2}_{\mathrm{LL}}$ (solid line) and $\mathbf{2}_{\mathrm{DD}}$ (dashed line) in the aggregated state, prepared by the sonication of each solution in MCH $\left(4 \times 10^{-5} \mathrm{M}\right)$ for $10 \mathrm{~min}$.

Both monomeric diamide $\mathbf{1}$ and dimeric diamide $\mathbf{2}_{\mathbf{L L}}$ exhibited thermal hysteresis when cycled through the assembly and disassembly processes. The absorbance change of a solution of 1 in $\mathrm{MCH}$ was monitored during heating from 283 $\mathrm{K}$ to $363 \mathrm{~K}$ at a rate of $1 \mathrm{~K} \mathrm{~min}^{-1}$ and exhibited a non-sigmoidal transition from the aggregated state to the non-aggregated state with a critical (elongation) temperature $\left(T_{\mathrm{e}}\right)$ of $308 \mathrm{~K}$ (Figure 6a). During cooling of the resultant solution of molecularly dispersed 1, initiation of supramolecular polymerization occurred not at $T_{\mathrm{e}}=308 \mathrm{~K}$, but instead at $T_{\mathrm{e}}{ }^{\prime}=288 \mathrm{~K}$. Notably, the dimeric diamide $\mathbf{2}_{\mathbf{L L}}$ showed a more drastic thermal hysteresis; the difference between the critical temperatures $\left(\Delta T_{\mathrm{e}}\right.$ $\left.=T_{\mathrm{e}}-T_{\mathrm{e}}{ }^{\prime}\right)$ for $\mathbf{2}_{\mathbf{L L}}$ was much greater than the $\Delta T_{\mathrm{e}}$ value of $20 \mathrm{~K}$ 
observed for $\mathbf{1}$. The $T_{\mathrm{e}}$ value for $\mathbf{2}_{\mathbf{L L}}$ upon heating was $353 \mathrm{~K}$ (Figure 6b), demonstrating the high thermal stability of $\mathbf{2}_{\mathbf{L L}}$ in the aggregated state, likely due to the multiple intermolecular interactions between the diamide moieties as well as the pyrene moieties in the supramolecular polymer chain. During cooling of the resultant solution of $\mathbf{2}_{\mathrm{LL}}$, spontaneous supramolecular polymerization was inhibited even at $283 \mathrm{~K}$. The high stability of the non-aggregated state should be attributed to the multiple intramolecular hydrogen bonds in the metastable 14membered-ring folded state.
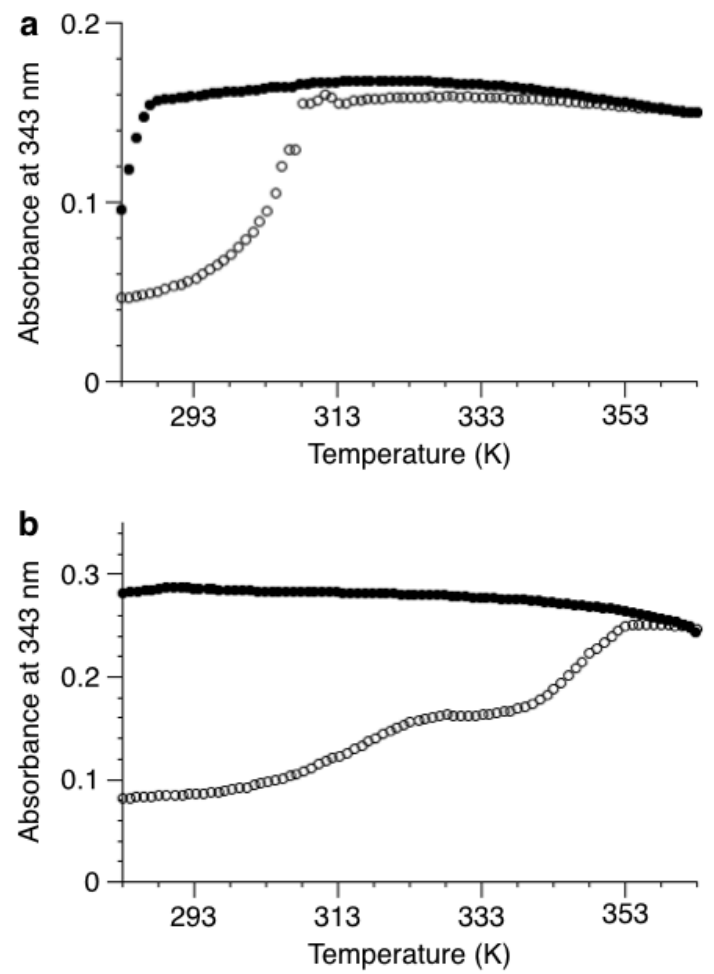

Figure 6. Thermal hysteresis observed during slow $\left(1 \mathrm{~K} \mathrm{~min}{ }^{-1}\right)$ cooling ( $\bullet$ ) and heating ( $\circ$ ) of (a) $\mathbf{1}$ and (b) $\mathbf{2}_{\mathrm{LL}}$ by monitoring the absorbance changes at $343 \mathrm{~nm}$ in $\mathrm{MCH}\left(4 \times 10^{-5} \mathrm{M}\right)$.

The dimeric diamide $\mathbf{2}_{\mathbf{L L}}$ showed another characteristic behavior during the heating process. The absorbance change observed upon heating aggregated $\mathbf{2}_{\mathbf{L L}}$ was not fitted using the cooperative nucleation-elongation model due to the existence of another transition at approximately $332 \mathrm{~K}$. This transition accompanied the emergence of $\mathrm{CD}$ absorption bands with negative and positive maxima at 336 and $347 \mathrm{~nm}$, respectively (Figure S9a). Since the temperature at which the CD absorption bands appeared was independent of the total concentration of $\mathbf{2}_{\mathrm{LL}}$ (Figure $\mathrm{S} 9 \mathrm{~b}$ ), this transition is likely related to the rearrangement of the orientation of the pyrene moieties in the aggregated state.

Seeded polymerization. The initiation step of the helical supramolecular polymerization of $\mathbf{2}_{\mathrm{LL}}$ could be kinetically regulated via a seeding method. A solution of seeds was prepared by sonication of a hot solution of $\mathbf{2}_{\mathbf{L L}}$ in $\mathrm{MCH}$ for 10 min with a water bath below $283 \mathrm{~K}$. Fast cooling of a solution of $\mathbf{2}_{\mathbf{L L}}$ in $\mathrm{MCH}$ from $363 \mathrm{~K}$ to $294 \mathrm{~K}$ gave a solution of kinetically trapped $\mathbf{2}_{\mathbf{L L}}$, to which the seed solution was added. The time course of the conversion of $\mathbf{2}_{\mathbf{L L}}$ from the non- aggregated to the aggregated state was monitored via the absorbance change at $343 \mathrm{~nm}$ in the UV absorption spectra at $294 \mathrm{~K}$. Upon the addition of the seed solution, supramolecular polymerization was initiated without a lag time (Figure 7a). When pure $\mathrm{MCH}$ was added to the fast-cooled solution of $\mathbf{2}_{\mathbf{L L}}$ in $\mathrm{MCH}$ as a control experiment, the metastable folded state remained unchanged for more than $1 \mathrm{~h}$. The elongation of the nanofibers was confirmed by TEM observation of the sample prepared from the seeded solution (Figures $7 \mathrm{~b}$ and $7 \mathrm{c}$ ). These results demonstrated that the supramolecular polymerization was indeed initiated by the addition of the seeds.
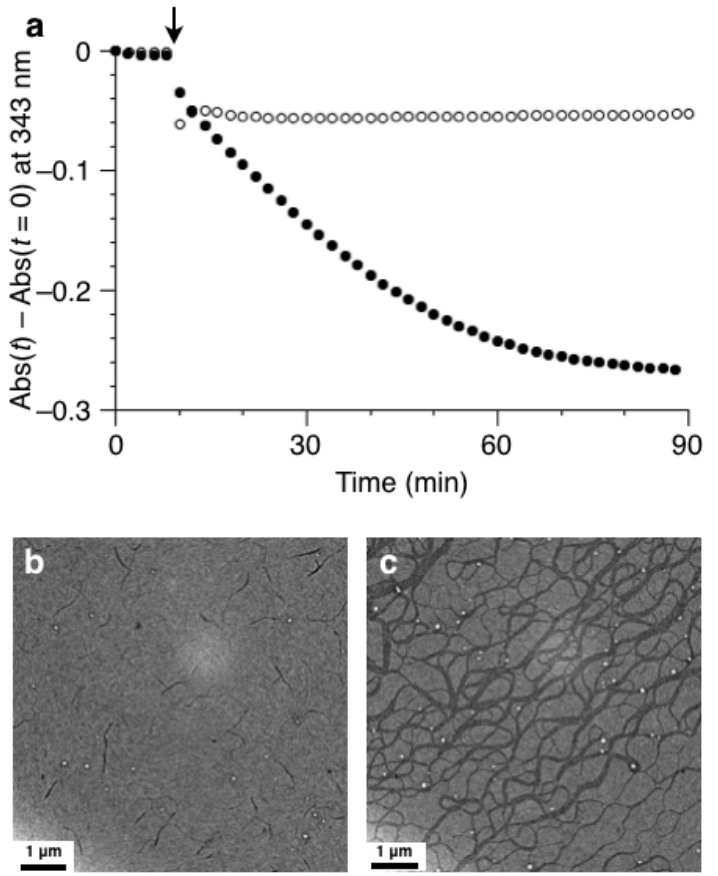

Figure 7. (a) Time-dependent change of the absorbance at $343 \mathrm{~nm}$ before and after addition of either a $\mathrm{MCH}$ solution of $\mathbf{2}_{\mathbf{L L}}$ seeds $\left(0.03 \mathrm{~mL} ; 5 \times 10^{-5} \mathrm{M}\right.$; closed circles $)$ or pure $\mathrm{MCH}(0.03 \mathrm{~mL}$; open circles) to $0.3 \mathrm{~mL}$ of $2_{\mathrm{LL}}$ in $\mathrm{MCH}\left(5 \times 10^{-5} \mathrm{M}\right)$ at the time indicated by the arrow, and TEM images of (b) $\mathbf{2}_{\mathbf{L L}}$ seeds and (c) the aggregates obtained after the seeded polymerization.

Furthermore, we elucidated the effect of the chirality of the diamide moieties on the seeded helical supramolecular polymerization. Using the procedure described above, solutions of $\mathbf{2}_{\mathrm{LL}}$ and $\mathbf{2}_{\mathrm{DD}}$ seeds were prepared separately and added sequentially to a fast-cooled solution of $\mathbf{2}_{\mathbf{L L}}$ in $\mathrm{MCH}$, in which $\mathbf{2}_{\mathbf{L L}}$ was present as the metastable folded state. A timedependent UV absorption spectroscopic study showed that no change occurred upon the addition of $\mathbf{2}_{\mathrm{DD}}$ seeds, while the supramolecular polymerization of $\mathbf{2}_{\mathbf{L L}}$ was initiated immediately after the subsequent addition of $\mathbf{2}_{\mathbf{L L}}$ seeds (Figure S10). Such self-sorting behavior was also observed in spontaneous supramolecular polymerization from a mixture of $\mathbf{2}_{\text {DD }}$ and $\mathbf{2}_{\mathbf{L L}}$ in the metastable folded state. CD spectra were measured for $\mathrm{MCH}$ solutions containing various molar ratios of $\mathbf{2}_{\mathrm{DD}}$ and $\mathbf{2}_{\mathrm{LL}}$ in a total concentration of $4 \times 10^{-5} \mathrm{M}$. A plot of the $\mathrm{CD}$ intensity at $357 \mathrm{~nm}$ as a function of the mole fraction of $\mathbf{2}_{\mathbf{L L}}$ exhibited an approximately linear relationship, suggesting that $\mathbf{2}_{\mathrm{DD}}$ and $\mathbf{2}_{\mathbf{L L}}$ were self-sorted in the aggregated state (Figure S11). The addition of $\mathbf{2}_{\mathbf{L L}}$ seeds followed by $\mathbf{2}_{\mathrm{DD}}$ seeds to a $1: 1$ 
mixed solution of $\mathbf{2}_{\mathrm{DD}}$ and $\mathbf{2}_{\mathrm{LL}}$ in the metastable folded state in a stepwise manner resulted in stepwise decreases in the absorbance at $343 \mathrm{~nm}$ (Figure 8). The rate of the supramolecular polymerization in the latter process was slightly lower, likely due to the dilution of the non-aggregated $\mathbf{2}_{\mathbf{D D}}$ by the addition of the $\mathrm{MCH}$ solution of $\mathbf{2}_{\mathrm{LL}}$ seeds.

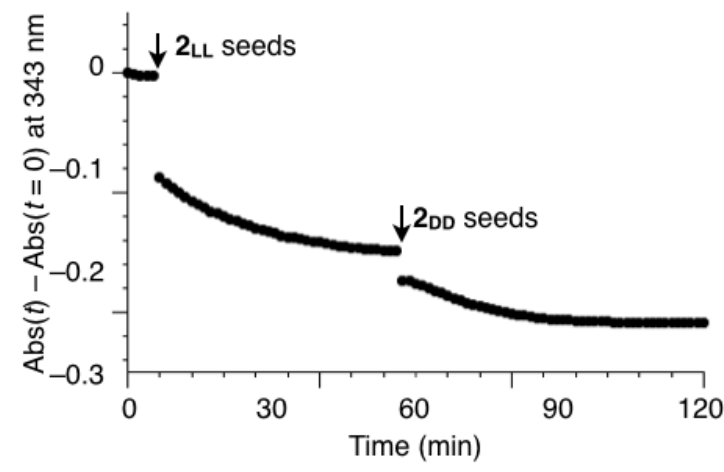

Figure 8. Time-dependent change in the absorbance at $343 \mathrm{~nm}$ before and after the addition of a MCH solution of $\mathbf{2}_{\mathbf{L L}}$ seeds $(0.1$ $\left.\mathrm{mL} ; 5 \times 10^{-5} \mathrm{M}\right)$ followed by the addition of $\mathbf{2}_{\mathrm{DD}}$ seeds $(0.1 \mathrm{~mL} ; 5$ $\times 10^{-5} \mathrm{M}$ ) to $0.2 \mathrm{~mL}$ of molecularly dispersed $\mathbf{2}_{\mathbf{L L}}$ and $\mathbf{2}_{\mathrm{DD}}$ in $\mathrm{MCH}$ $\left(2.5 \times 10^{-5} \mathrm{M}\right.$ each $)$ at the times indicated by the arrows.

Finally, taking advantage of the kinetic stability of $\mathbf{2}_{\mathbf{L L}}$ in the folded state, we performed seed-initiated supramolecular polymerizations in a microflow channel. Microfluidic mixing has been used to kinetically control the self-assembly processes of $\pi$-conjugated molecules. ${ }^{39-41}$ By modulating the flow rate and the geometry of the microchip, the diffusion zone in which the metastable monomers met the seeds could be precisely defined. The chip geometry we used is shown in Figure 9a. A $\mathrm{MCH}$ solution of $\mathbf{2}_{\mathrm{LL}}$ seeds and one of non-aggregated $\mathbf{2}_{\mathbf{L L}}$ were flowed into the microflow channel from three glass syringes controlled by syringe pumps. When only the non-aggregated $\mathbf{2}_{\mathrm{LL}}$ solution was flowed, the absorption spectrum of the solution collected from the outlet of the microchip was identical to that of molecularly dispersed $\mathbf{2}_{\mathbf{L L}}$ (Figure $9 \mathrm{~b}$, dashed line). In contrast, when a solution of $\mathbf{2}_{\mathbf{L L}}$ seeds was flowed from the middle inlet and mixed with a solution of a folded form of $\mathbf{2}_{\mathbf{L L}}$, which was flowed from the side inlets, the solution obtained from the outlet mainly contained elongated $\mathbf{2}_{\mathbf{L L}}$ aggregates, as was evident from its UV spectrum (Figure 9b, solid line) and TEM observations (Figure S12a). The resultant solution showed no spectral change over an hour, suggesting that the metastable folded form of $\mathbf{2}_{\mathbf{L L}}$ efficiently interacted with the seeds to produce supramolecular polymers in a continuously flowing stream (Figure S12b). These results demonstrated that the kinetic and thermodynamic stabilities of dimeric diamide $\mathbf{2}_{\mathbf{L L}}$ in the non-aggregated and aggregated forms, respectively, are appropriate for achieving seeded supramolecular polymerization under the microfluidic mixing conditions.

\section{CONCLUSIONS}

In conclusion, we have elucidated the folding and assembly behavior of a cystine-based dimeric diamide in nonpolar solution. Compared with the alanine-based monomeric diamide, the dimeric diamide shows several unique features: (1) Its spontaneous self-assembly upon cooling is efficiently retarded due to the formation of a folded 14-membered ring a
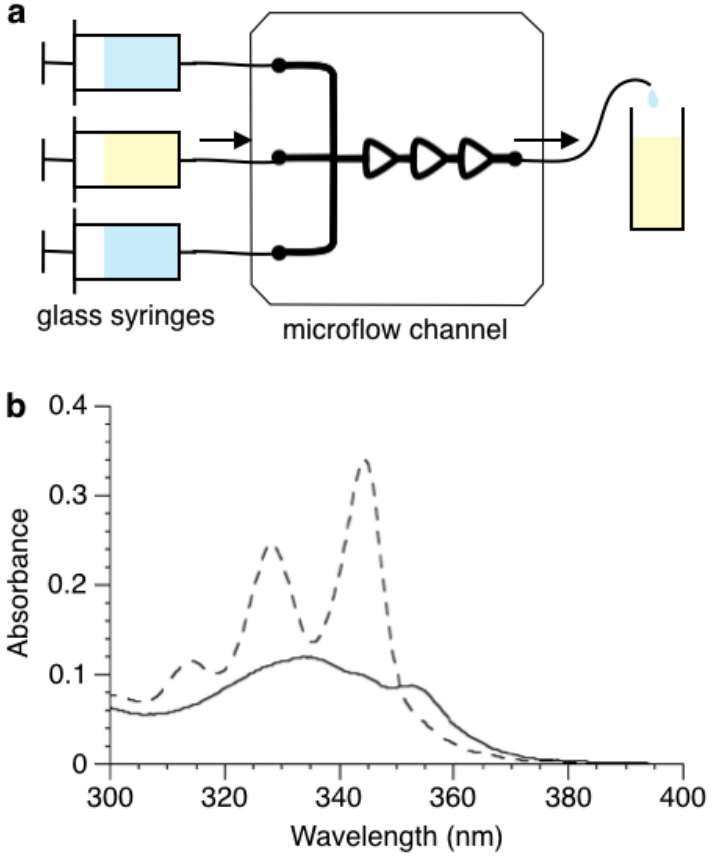

Figure 9. (a) Schematic representation of the microflow system and (b) UV absorption spectra of $\mathbf{2}_{\mathbf{L L}}$ in the monomeric state (dashed line), which was obtained after flowing a solution of metastable monomeric $\mathbf{2}_{\mathbf{L L}}$ through the microflow channel, and of $\mathbf{2}_{\mathbf{L L}}$ in the aggregated state (solid line), which was obtained after seeded polymerization under microfluidic mixing.

conformation with two intramolecular hydrogen bonds between the two diamide units; (2) the metastable folded form eventually transforms into the thermodynamically stable supramolecular polymers, which show increased thermal stability; and (3) the chirality of the dimeric diamide determines the helical arrangement of the pyrene units in the aggregated state. The improvement of the kinetic stability in the non-aggregated state enabled us to extend the seed-initiated supramolecular polymerization to microfluidic mixing conditions. Furthermore, we confirmed that the addition of an enantiomerically excess amount of L-cysteine-based diamide dimers did not suppress the chiral expression of D-cysteine-based dimeric diamides in the aggregated state. Taking advantage of the self-sorting behavior, two-step supramolecular polymerization from a racemic mixture of L-cysteine- and D-cysteine-based dimeric diamides was achieved upon stepwise addition of the corresponding seeds.

The use of an amino acid is advantageous because the $\mathrm{NH}_{2}-$ and $\mathrm{COOH}$-termini are easily functionalized by various $\pi$ conjugated skeletons through amidation reactions. Thus, the dimeric structure of the amino-acid-based diamide should have significant potential as a building block for engineering supramolecular assemblies from both thermodynamic and kinetic points of view. The molecular design of the metastable monomers, as well as the developed methodology for seeded polymerization, would enable us to produce well-defined nanostructures for various applications in the areas of organic electronics and photonics. To this end, it is required to precisely tune the kinetic stability of the folded diamide not only low- 
polarity solvents, but also in aqueous media. ${ }^{42} \mathrm{~A}$ further study along these lines is currently in progress in our laboratory.

\section{ASSOCIATED CONTENT}

\section{Supporting Information.}

Analyses of self-assembly behaviors of $\mathbf{1}$ and $\mathbf{2}_{\mathrm{LL}}$, AFM studies of $\mathbf{1}$ and $\mathbf{2}_{\mathbf{L L}}$ aggregate samples. This material is available free of charge via the Internet at http://pubs.acs.org.

\section{AUTHOR INFORMATION}

\section{Corresponding Author}

ogi.soichiro@chem.nagoya-u.ac.jp, yamaguchi@chem.nagoyau.ac.jp

\section{ORCID}

Soichiro Ogi: 0000-0001-5758-2281

Shigehiro Yamaguchi: 0000-0003-0072-8969

\section{Author Contributions}

S.O. conceived and directed the project. T.A. carried out most of the experimental work. M.K. synthesized compound 1. S.O. and S.Y. co-wrote the manuscript. S.O., T.A., K.M., and S.Y. discussed the results and commented on the manuscript.

\section{Notes}

The authors declare no competing financial interest.

\section{ACKNOWLEDGMENT}

The authors are grateful to Prof. Hiroaki and Dr Shigemitsu for kindly supporting the 2D NMR studies, and to Dr Oyama for the mass spectroscopic anaysis. The authors thank to Prof. Yanai and Prof. Fujimoto for helpful discussions related to the DFT calculation, and Prof. Numata and Ms. Kanzaki (Kyoto City Univ.) for helpful advice related to the microflow systems. This work was supported by KAKENHI grants (19K05419, 18H03909, and 18H05261) from the Japan Society for the Promotion of Science (JSPS). A part of this research is based on the Cooperative Research Project of Research Center for Biomedical Engineering, Tokyo Medical and Dental University.

\section{REFERENCES}

1. Hamley, I. W. The Amyloid Beta Peptide: A Chemist's Perspective. Role in Alzheimer's and Fibrillization. Chem Rev 2012, $112,5147-5192$.

2. Knowles, T. P. J.; Mezzenga, R. Amyloid Fibrils as Building Blocks for Natural and Artificial Functional Materials. Adv. Mater. 2016, 28, 6546-6561.

3. Friedhoff, P.; von Bergen, M.; Mandelkow, E.-M.; Davies, P.; Mandelkow, E. A Nucleated Assembly Mechanism of Alzheimer Paired Helical Filaments. Proc. Natl. Acad. Sci. U. S. A. 1998, 95, 15712-15717.

4. Hartl, F. U.; Hayer-Hartl, M. Molecular Chaperones in the Cytosol: From Nascent Chain to Folded Protein. Science 2002, 295, $1852-1858$

5. Gazit, E. The "Correctly Folded" State of Proteins: Is It a Metastable State? Angew. Chem. Int. Ed. 2002, 41, 257-259.

6. Dobson, C. M. Protein Folding and Misfolding. Nature 2003, 426, 884-890.

7. Röder, K.; Wales, D. J. Energy Landscapes for the Aggregation of A $\beta_{17-42 .}$. Am. Chem. Soc. 2018, 140, 4018-4027.

8. Adamcik, J.; Mezzenga, R. Amyloid Polymorphism in the Protein Folding and Aggregation Energy Landscape. Angew. Chem. Int. Ed. 2018, 57, 8370-8382.
9. Jarrett, J. T.; Lansbury Jr., P. T. Seeding "OneDimensional Crystallization" of Amyloid: A Pathogenic Mechanism in Alzheimer's Disease and Scrapie? Cell 1993, 73, 1055-1058.

10. Wetzel, R. Kinetics and Thermodynamics of Amyloid Fibril Assembly. Acc. Chem. Res. 2006, 39, 671-679.

11. Hu, X.; Crick, S. L.; Bu, G.; Frieden, C.; Pappu, R. V.; Lee, J.-M. Amyloid Seeds Formed by Cellular Uptake, Concentration, and Aggregation of the Amyloid-Beta Peptide. Proc. Natl. Acad. Sci. U. S. A. 2009, 106, 20324-20329.

12. Cohen, S. I.; Linse, S.; Luheshi, L. M.; Hellstrand, E.; White, D. A.; Rajah, L.; Otzen, D. E.; Vendruscolo, M.; Dobson, C. M.; Knowles, T. P. J. Proliferation of Amyloid- $\beta 42$ Aggregates Occurs through a Secondary Nucleation Mechanism. Proc. Natl. Acad. Sci. U. S. A. 2013, 110, 9758-9763.

13. Buell, A. K.; Galvagnion, C.; Gaspar, R.; Sparr, E.; Vendruscolo, M.; Knowles, T. P. J.; Linse, S.; Dobson, C. M. Solution Conditions Determine the Relative Importance of Nucleation and Growth Processes in $\alpha$-Synuclein Aggregation. Proc. Natl. Acad. Sci. U. S. A. 2014, 111, 7671-7676.

14. van der Zwaag, D.; de Greef, T. F. A.; Meijer, E. W. Programmable Supramolecular Polymerizations. Angew. Chem., Int. Ed. 2015, 54, 8334-8336.

15. Matern, J.; Dorca, Y.; Sánchez, L.; Fernández, G. Revising Complex Supramolecular Polymerization under Kinetic and Thermodynamic Control. Angew. Chem. Int. Ed. 2019, 58, 1673016740 .

16. Wehner, M.; Würthner, F. Supramolecular Polymerization through Kinetic Pathway Control and Living Chain Growth. Nature Reviews Chemistry 2020, 4, 38-53.

17. Ogi, S.; Sugiyasu, K.; Manna, S.; Samitsu, S.; Takeuchi, M. Living Supramolecular Polymerization Realized through a Biomimetic Approach. Nat. Chem. 2014, 6, 188-195.

18. Pal, A.; Malakoutikhah, M.; Leonetti, G.; Tezcan, M.; Colomb-Delsuc, M.; Nguyen, V. D.; van der Gucht, J.; Otto, S. Controlling the Structure and Length of Self-Synthesizing Supramolecular Polymers through Nucleated Growth and Disassembly. Angew. Chem., Int. Ed. 2015, 54, 7852-7856.

19. Robinson, M. E.; Lunn, D. J.; Nazemi, A.; Whittell, G. R.; De Cola, L.; Manners, I. Length Control of Supramolecular Polymeric Nanofibers based on Stacked Planar Platinum(II) Complexes by Seeded-Growth. Chem Commun 2015, 51, 1592116924.

20. Endo, M.; Fukui, T.; Jung, S. H.; Yagai, S.; Takeuchi, M.; Sugiyasu, K. Photoregulated Living Supramolecular Polymerization Established by Combining Energy Landscapes of Photoisomerization and Nucleation-Elongation Processes. J. Am. Chem. Soc. 2016, 138, 14347-14353.

21. Fukui, T.; Kawai, S.; Fujinuma, S.; Matsushita, Y.; Yasuda, T.; Sakurai, T.; Seki, S.; Takeuchi, M.; Sugiyasu, K. Control over Differentiation of a Metastable Supramolecular Assembly in One and Two Dimensions. Nat. Chem. 2017, 9, 493-499.

22. Zhang, W.; Jin, W.; Fukushima, T.; Saeki, A.; Seki, S.; Aida, T. Supramolecular Linear Heterojunction Composed of Graphite-Like Semiconducting Nanotubular Segments. Science 2011, $334,340-343$.

23. Zhang, W.; Jin, W.; Fukushima, T.; Mori, T.; Aida, T. Helix Sense-Selective Supramolecular Polymerization Seeded by a One-Handed Helical Polymeric Assembly. J. Am. Chem. Soc. 2015, $137,13792-13795$.

24. Ma, X.; Zhang, Y.; Zhang, Y.; Liu, Y.; Che, Y.; Zhao, J. Fabrication of Chiral-Selective Nanotubular Heterojunctions through Living Supramolecular Polymerization. Angew. Chem. Int. Ed. 2016, 55, 9539-9543.

25. Jung, S. H.; Bochicchio, D.; Pavan, G. M.; Takeuchi, M.; Sugiyasu, K. A Block Supramolecular Polymer and Its Kinetically Enhanced Stability. J. Am. Chem. Soc. 2018, 140, 10570-10577.

26. Liu, Y.; Gong, Y. J.; Guo, Y. X.; Xiong, W.; Zhang, Y. F.; Zhao, J. C.; Che, Y. K.; Manners, I. Emergent Self-Assembly 
Pathways to Multidimensional Hierarchical Assemblies using a Hetero-Seeding Approach. Chem. - Eur. J. 2019, 25, 13484-13490.

27. Wagner, W.; Wehner, M.; Stepanenko, V.; Würthner, F. Supramolecular Block Copolymers by Seeded Living Polymerization of Perylene Bisimides. J. Am. Chem. Soc. 2019, 141, 12044-12054.

28. Sarkar, A.; Sasmal, R.; Empereur-mot, C.; Bochicchio, D.; Kompella, S. V. K.; Sharma, K.; Dhiman, S.; Sundaram, B.; Agasti, S. S.; Pavan, G. M.; George, S. J. Self-Sorted, Random, and Block Supramolecular Copolymers via Sequence Controlled, Multicomponent Self-Assembly. J. Am. Chem. Soc. 2020, 142, 76067617.

29. Wehner, M.; Röhr, M. I. S.; Bühler, M.; Stepanenko, V.; Wagner, W.; Würthner, F. Supramolecular Polymorphism in OneDimensional Self-Assembly by Kinetic Pathway Control. J. Am. Chem. Soc. 2019, 141, 6092-6107.

30. Kang, J.; Miyajima, D.; Mori, T.; Inoue, Y.; Itoh, Y.; Aida, T. Noncovalent Assembly. A Rational Strategy for the Realization of Chain-Growth Supramolecular Polymerization. Science 2015, 347, 646-651.

31. Ogi, S.; Stepanenko, V.; Sugiyasu, K.; Takeuchi, M.; Würthner, F. Mechanism of Self-Assembly Process and Seeded Supramolecular Polymerization of Perylene Bisimide Organogelator. $J$ Am. Chem. Soc. 2015, 137, 3300-3307.

32. Ogi, S.; Stepanenko, V.; Thein, J.; Würthner, F. Impact of Alkyl Spacer Length on Aggregation Pathways in Kinetically Controlled Supramolecular Polymerization. J. Am. Chem. Soc. 2016, $138,670-678$.

33. Pal, D. S.; Kar, H.; Ghosh, S. Controllable Supramolecular Polymerization via a Chain-Growth Mechanism. Chem. Commun. 2018, 54, 928-931.

34. Greciano, E. E.; Sánchez, L. Seeded Supramolecular Polymerization in a Three-Domain Self-Assembly of an N-Annulated Perylenetetracarboxamide. Chem. - Eur. J. 2016, 22, 13724-13730.

35. Valera, J. S.; Gómez, R.; Sánchez, L. Tunable Energy Landscapes to Control Pathway Complexity in Self-Assembled N-
Heterotriangulenes: Living and Seeded Supramolecular Polymerization. Small 2018, 14, 1702437.

36. Greciano, E. E.; Matarranz, B.; Sánchez, L. Pathway Complexity Versus Hierarchical Self-Assembly in N-Annulated Perylenes: Structural Effects in Seeded Supramolecular Polymerization. Angew. Chem., Int. Ed. 2018, 57, 4697-4701.

37. Wang, H.; Zhang, Y.; Chen, Y.; Pan, H.; Ren, X.; Chen, Z. Living Supramolecular Polymerization of an Aza-BODIPY Dye Controlled by a Hydrogen-Bond-Accepting Triazole Unit Introduced by Click Chemistry. Angew. Chem., Int. Ed. 2020, 59, 5185-5192.

38. Ogi, S.; Matsumoto, K.; Yamaguchi, S. Seeded Polymerization through the Interplay of Folding and Aggregation of an Amino-Acid-Based Diamide. Angew. Chem., Int. Ed. 2018, 57, 2339 2343 .

39. Numata, M. Supramolecular Chemistry in Microflow Fields: Toward a New Material World of Precise Kinetic Control. Chem. - Asian J. 2015, 10, 2574-2588.

40. Sevim, S.; Sorrenti, A.; Franco, C.; Furukawa, S.; Pané, S.; deMello, A. J.; Puigmartí-Luis, J. Self-Assembled Materials and Supramolecular Chemistry within Microfluidic Environments: From Common Thermodynamic States to Non-Equilibrium Structures. Chem. Soc. Rev. 2018, 47, 3788-3803.

41. Kanzaki, C.; Nakadozono, T.; Numata, M. Creation of Discrete 1D Microstructures: Directional Dissociation from the Ends of a Metastable Supramolecular Polymer. ChemPlusChem 2019, 85, 74-78.

42. Ogi, S.; Fukaya, N.; Arifin; Skjelstad, B. B.; Hijikata, Y.; Yamaguchi, S. Seeded Polymerization of an Amide-Functionalized Diketopyrrolopyrrole Dye in Aqueous Media. Chem. - Eur. J. 2019, 25, 7303-7307. 
Table of Contents Graphic

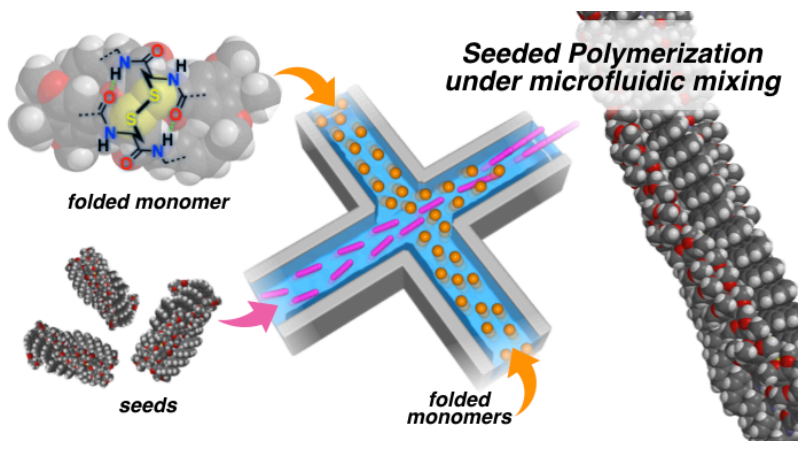

